\title{
Expression of miR-542-3p in osteosarcoma with miRNA microarray data, and its potential signaling pathways
}

\author{
ZHEN LI ${ }^{1,2}$, JIAN-NI YAO $^{3}$, WEN-TING HUANG ${ }^{4}$, RONG-QUAN HE ${ }^{5}$, \\ $\mathrm{JIE} \mathrm{MA}^{5}$, GANG CHEN $^{4}$ and QING-JUN WEI ${ }^{1,2}$ \\ ${ }^{1}$ Department of Orthopedic Surgery, The First Affiliated Hospital of Guangxi Medical University; \\ ${ }^{2}$ Collaborative Innovation Center of Guangxi Biological Medicine, Guangxi Medical University; \\ ${ }^{3}$ First Clinical Medical College of Guangxi Medical University; Departments of ${ }^{4}$ Pathology and ${ }^{5}$ Medical Oncology, \\ The First Affiliated Hospital of Guangxi Medical University, Nanning, Guangxi Zhuang Autonomous Region 530021, P.R. China
}

Received July 9, 2018; Accepted November 15, 2018

DOI: $10.3892 / \mathrm{mmr} .2018 .9761$

\begin{abstract}
Osteosarcoma (OS) is the most common pediatric primary bone tumor, with high malignancy rates and a poor prognosis following metastasis. At present, the role of microRNA (miR)-542-3p in OS remains to be elucidated. The purpose of the present study was to investigate the expression level of miR-542-3p in OS, and its potential molecular mechanisms, via a bioinformatics analysis. First, the expression of miR-542-3p in OS based on the continuous variables of the Gene Expression Omnibus database and PubMed was studied. Subsequently, the potential target genes of miR-542-3p were predicted using gene expression profiles and bioinformatics software. On the basis of the Database for Annotation, Visualization and Integrated Discovery, version 6.8, a study of gene ontology (GO) enrichment and the Kyoto Encyclopedia of Genes and Genomes (KEGG) pathway knowledge base was conducted to explore the biological value of miR-542-3p in OS. Finally, the protein-protein interaction (PPI) network was completed using the STRING database. The expression of miR-542-3p in OS was revealed to be significantly higher compared with that in normal tissue. In total, 1,036 target genes of miR-542-3p were obtained. The results of the GO enrichment analysis revealed that the significant terms were 'bone development', 'cell cycle arrest' and 'intracellular signal transduction'. The results of the KEGG analysis revealed the highlighted pathways that were targeted to miR-542-3p, including the sphingolipid signaling pathway $\left(\mathrm{P}=3.91 \times 10^{-5}\right)$, the phosphoinositide 3-kinase (PI3K)-AKT serine/threonine kinase (AKT) signaling pathway $\left(\mathrm{P}=3.17 \times 10^{-5}\right)$ and the insulin
\end{abstract}

Correspondence to: Professor Qing-Jun Wei, Department of Orthopedic Surgery, The First Affiliated Hospital of Guangxi Medical University, 6 Shuangyong Road, Nanning, Guangxi Zhuang Autonomous Region 530021, P.R. China

E-mail: weiqingjungxnn@163.com

Key words: osteosarcoma, microRNA-542-3p, microarray data, protein-protein interaction, signaling pathways signaling pathway $\left(\mathrm{P}=1.04 \times 10^{-5}\right)$. The PPI network revealed eight hub genes: Ubiquitin-60S ribosomal protein L40, Ras-related C3 botulinum toxin substrate, mitogen-activated protein kinase 1, epidermal growth factor receptor, cystic fibrosis transmembrane conductance regulator, PI3K regulatory subunit $1, \mathrm{AKT} 1$, and actin-related protein $2 / 3$ complex subunit $1 \mathrm{~A}$, which may be the key target genes of miR-542-3p in OS. Taken together, these results have demonstrated that miR-542-3p was overexpressed in OS. The potential target genes and biological functions of miR-542-3p may provide novel insights into the differentially expressed genes that are involved in OS.

\section{Introduction}

OS is the most common pediatric primary malignant bone tumor. The 5-year survival rates for children (0-14 years old) and adolescents (15-19 years old) are 69.5 and $63.4 \%$, respectively (1), whereas for metastatic patients, it is only $20-30 \%$ (2). At present, the pathogenesis and etiology of OS remain to be fully elucidated. A strategy that was implemented to determine treatment based on the histological response did not lead to any improvement in the survival rate (3). Early diagnosis and the improvement of therapeutic strategies are therefore urgently required.

MicroRNAs (miRNAs) are single-stranded RNA species that are highly conserved, between 20 and 24 nucleotides in length, and mature miRNAs are able to inhibit the translation and degradation of mRNAs by binding to their 3'-untranslated regions (UTRs) (4). Researchers have identified $>2,000$ miRNAs in Homo sapiens, and one-third of mRNAs are hypothesized to be co-regulated in the genome (5). At present, miRNAs have been demonstrated to be widely involved in physiological and pathological processes associated with cancer, including the differentiation, proliferation and apoptosis of cancer cells (6). Recently, increasing evidence has indicated that miRNAs are closely associated with the development and metastasis of OS; for example, miRNA (miR)-34a in OS targets the Notch pathway and causes Notch1 downregulation, resulting in cell cycle arrest and apoptosis $(7,8)$. The study of target genes and gene pathways of miRNAs is conducive to 
revealing the molecular mechanisms underpinning the development and metastasis of OS. Researchers have demonstrated that $\mathrm{miR}-542-3 \mathrm{p}$ is implicated in the progression of various types of tumors, including neuroblastoma, gastric cancer, bladder cancer and astrocytoma, via targeted inhibition of angiopoietin-2 (9-12). A recent study verified that miR-542-3p is an oncogene of OS, which is able to enhance cell proliferation and migration at an overexpressed level in OS, and one of its target genes is VANGL planar cell polarity protein 2 (VANGL2) (13). However, the target gene(s) of miR-542-3p, and its underlying mechanism(s) in OS, remain unclear.

In the present study, the high expression level of miR-542-3p in OS, based on the continuous variables of the Gene Expression Omnibus (GEO) database and PubMed, was first confirmed. Subsequently, the potential target genes of miR-542-3p were identified using bioinformatics software and gene expression profiles. Using the Database for Annotation, Visualization and Integrated Discovery (DAVID), version 6.8, the biological value of miR-542-3p was identified using gene ontology (GO) enrichment and Kyoto Encyclopedia of Genes and Genomes (KEGG) pathway analyses. The top 10 results of the GO enrichment analysis were selected, and the KEGG results revealed the highlighted pathways of miR-542-3p. Taken together, the identification of the potential target genes and biological functions of miR-542-3p has provided novel insights into the role of differentially expressed genes (DEGs) in OS. miR-542-3p may therefore be a novel marker useful for diagnosis and treatment of patients with OS.

\section{Materials and methods}

Study of a comparison of the expression levels of miR-542-3p between patients with $O S$ and normal controls (NC). As presented in Fig. 1, miR-542-3p expression profiles of OS were searched from the GEO (https://www.ncbi.nlm. nih.gov/gds/?term=) and ArrayExpress (https://www.ebi. ac.uk/arrayexpress/) databases to identify the expression level of miR-542-3p in OS. The search strategy employed was as follows: [('bone' OR 'bones') AND ('sarcoma' OR 'sarcomas')] OR ('osteosarcoma' OR 'osteosarcomas') AND "Homo sapiens"] [porgn_txid9606]. In addition, the PubMed database (https://www.ncbi.nlm.nih.gov/pubmed/?term=) was also searched using the following retrieval strategy: ('microRNAs' [medical subject heading (MeSH) terms] OR 'microRNAs' (All fields) OR 'osteosarcoma' (All fields)], retrieving results from all studies published up to March 2018 to ensure that any relevant publications were not overlooked. Exclusion criteria were as follows: Only studies of miR-542-3p expression that involved a comparison being made between $\mathrm{OS}$ and $\mathrm{NC}$ were analyzed, and studies that were duplicates or lacked a sufficient sample size (i.e., the samples of each group were $<3$ ) were also excluded.

Qualifying OS gene expression profiles and identification of DEGs. To understand the biological role of target genes of miR-542-3p in OS, qualifying gene expression profiles from the GEO database were selected for further study. In order to obtain DEGs between OS and NC (normal bone or osteoblasts or mesenchymal stem cells), 14 expression profiles were selected for analysis, and the characteristics of the individual

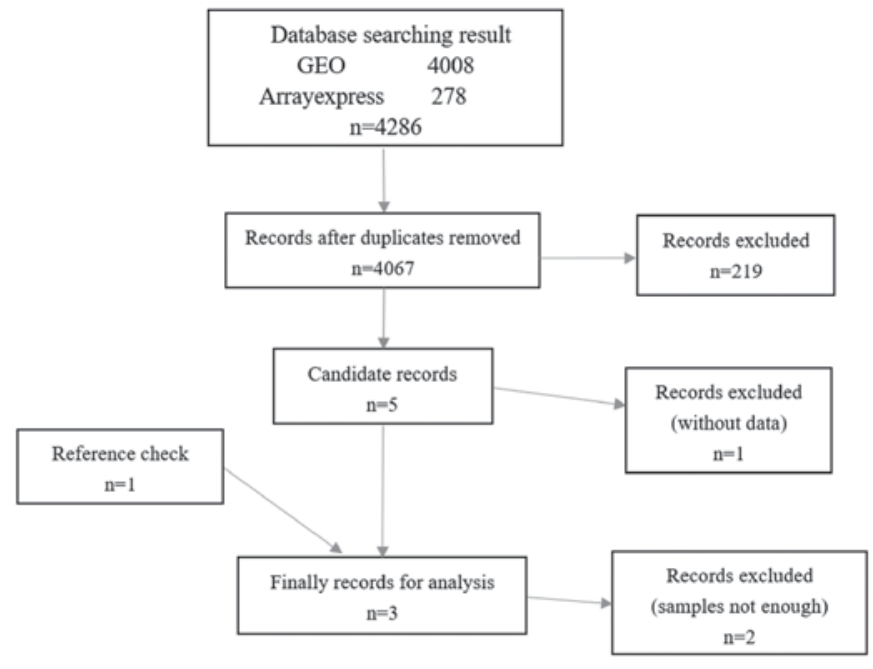

Figure 1. Flow chart indicating the identification of studies based on microRNA-542-3p expression values comparing between patients with osteosarcoma and normal controls. GEO, Gene Expression Omnibus.

studies are presented in Table I. The raw data file was uploaded to the online resource GEO2R (https://www.ncbi.nlm.nih. gov/geo/info/geo2r.html). The truncated standard, $\log \mathrm{FC}<0$, was used to determine the DEGs with statistical significance. Qualifying genes were classified as such if they appeared in no fewer than six expression profiles, and these were used for further study. A search was also performed for gene expression chips that were designed for the transfection of miRNA or miR-542-3p mimics. The expression profile GSE47363 (14), based on GPL10558 (Illumina HumanHT-12 v4.0 Expression BeadChip Support; Illumina, Inc., San Diego, CA, USA), was the only one that qualified. Subsequently, the OS gene expression profiles and the OS expression profile with the DEGs transfected with miR-542-3p were overlaid.

Prediction of target genes of miR-542-3p in OS based on MirWalk2.0. The miR-542-3p target genes were predicted based on miRWalk2.0, the comprehensive atlas of predicted and validated miRNA-target interactions (http://zmf.umm. uni-heidelberg.de/mirwalk2), using 12 predictive software packages, including DIANA-microT v4.0 (http://diana.imis. athena-innovation.gr/DianaTools/index.php?r=microtv4/index), DIANA-microT-CDS (http://diana.imis.athena-innovation. gr/DianaTools/index.php?r=microT_CDS/index),miRandarelease 2010 (http://www.microrna.org/microrna/getDownloads.do), mirBridge [(http://mirsystem.cgm.ntu.edu.tw/)), miRDB4.0 (http://mirdb.org/miRDB/download.html), miRmap (https://mirmap.ezlab.org/), miRNAMap (ftp://mirnamap. mbc.nctu.edu.tw/), PicTar2 (https://dorina.mdc-berlin. de/rbp_browser/download_hg19.html), PITA (https://genie. weizmann.ac.il/pubs/mir07/mir07_dyn_data.html)], RNA22v2 (https://cm.jefferson.edu/rna22/), RNAhybrid2.1 (https://bibiserv.cebitec.uni-bielefeld.de/rnahybrid/dl_pre-page. html) and Targetscan version 6.2 (http://www.targetscan. org/cgi-bin/targetscan/data_download.cgi?db=vert_61). The complete data were downloaded from the platform, and genes that featured in more than four of the predictive software packages were subsequently selected as putative target genes of miR-542-3p in OS. 
Table I. Characteristics of individual studies.

\begin{tabular}{|c|c|c|c|c|}
\hline GEO ID & $\begin{array}{l}\text { Sample count } \\
\text { (osteosarcoma/ } \\
\text { normal control) }\end{array}$ & Platform & Sample sources & Tissue \\
\hline GSE14359 & $18 / 2$ & $\begin{array}{l}\text { GPL96 [HG-U133A] Affymetrix Human } \\
\text { Genome U133A Array }\end{array}$ & in vivo & Bone, lung \\
\hline GSE12865 & $12 / 2$ & $\begin{array}{l}\text { GPL6244 [HuGene-1_0-st] Affymetrix } \\
\text { Human Gene 1.0 ST Array [transcript } \\
\text { (gene) version] }\end{array}$ & in vivo & Bone \\
\hline GSE11414 & $4 / 2$ & $\begin{array}{l}\text { GPL6244 [HuGene-1_0-st] Affymetrix } \\
\text { Human Gene 1.0 ST Array [transcript } \\
\text { (gene) version] }\end{array}$ & in vitro & Bone \\
\hline GSE42352 & $103 / 15$ & $\begin{array}{l}\text { GPL } 10295 \text { Illumina human- } 6 \text { v2.0 } \\
\text { expression beadchip (using nuIDs as } \\
\text { identifier) }\end{array}$ & in vivo/in vitro & Bone \\
\hline GSE36001 & $19 / 6$ & $\begin{array}{l}\text { GPL6102 Illumina human-6 v2.0 } \\
\text { expression beadchip }\end{array}$ & in vitro & Bone \\
\hline GSE32964 & $35 / 1$ & $\begin{array}{l}\text { GPL6947 Illumina HumanHT-12 } \\
\text { V3.0 expression beadchip }\end{array}$ & in vivo & Bone \\
\hline GSE68591 & $10 / 2$ & $\begin{array}{l}\text { GPL11028 [HuEx-1_0-st] Affymetrix } \\
\text { Human Exon 1.0 ST Array [HuEx-1_0-st-v2, } \\
\text { coreR3, A20071112, EP.cdf] }\end{array}$ & in vitro & Bone \\
\hline GSE70414 & $5 / 1$ & $\begin{array}{l}\text { GPL570 [HG-U133_Plus_2] Affymetrix } \\
\text { Human Genome U133 Plus 2.0 Array }\end{array}$ & in vitro & Bone \\
\hline GSE56001 & $3 / 5$ & $\begin{array}{l}\text { GPL10558 Illumina HumanHT-12 V4.0 } \\
\text { expression beadchip }\end{array}$ & in vivo & Bone \\
\hline GSE39262 & $10 / 1$ & $\begin{array}{l}\text { GPL96 [HG-U133A] Affymetrix Human } \\
\text { Genome U133A Array }\end{array}$ & in vitro & Bone \\
\hline GSE28424 & $19 / 4$ & $\begin{array}{l}\text { GPL } 13376 \text { Illumina HumanWG-6 v2.0 } \\
\text { expression beadchip }\end{array}$ & in vitro & Bone \\
\hline GSE14789 & $3 / 1$ & $\begin{array}{l}\text { GPL571 [HG-U133A_2] Affymetrix } \\
\text { Human Genome U133A 2.0 Array }\end{array}$ & in vitro & Bone \\
\hline GSE5045 & $5 / 2$ & $\begin{array}{l}\text { GPL1120 SuperArray GEArray Q } \\
\text { Series Human Angiogenesis Gene Array }\end{array}$ & in vivo & Bone \\
\hline GSE5045 & $5 / 3$ & $\begin{array}{l}\text { GPL1133 SuperArray GEArray Q Series } \\
\text { Human Tumor Metastasis Gene Array }\end{array}$ & in vivo & Bone \\
\hline
\end{tabular}

GEO, Gene Expression Omnibus.

Potential target genes of miR-542-3p in OS. As previously mentioned, DEGs in OS gene expression microarrays and predicted target genes of miR-542-3p based on miRWalk2.0 were collected. These genes were subsequently overlaid, and 1,035 potential target genes of miR-542-3p in OS were finally obtained. In addition, target genes of miR-542-3p in OS reported in the literature were collected. The inclusion criteria were as follows: i) miR-542-3p target genes that were identified in OS; and ii) a dual luciferase reporter assay or RNA binding protein immunoprecipitation assay had been used for verification of the target genes.

Bioinformatics analyses of the potential target genes. DAVID version 6.8 was used to perform GO enrichment and KEGG pathway analyses of the potential target genes of miR-542-3p in OS, and the threshold value was set at $\mathrm{P}<0.05$. In addition, in order to examine the interrelationships between these genes, protein-protein interaction (PPI) network analysis was performed using the STRING database (https://string-db.org/). Genes that were revealed to be hub genes were designated as the key target genes of miR-542-3p in OS. The correlation between proteins was evaluated using a reliability scoring threshold $>0.9$.

Statistical analysis. The expression data of miR-542-3p, comparing between $\mathrm{OS}$ and $\mathrm{NC}$, was analyzed using the software package Stata (version 12.0; StataCorp LP, College Station, TX, USA). Continuous variable analysis was performed on three studies. The expression level of miR-542-3p in OS was evaluated using a fixed-effects model 
Table II. Results of continuous variable analysis for identifying miR-542-3p expression level in osteosarcoma.

\begin{tabular}{lcccccc}
\hline & & \multicolumn{2}{c}{$\mathrm{n}$} & & \multicolumn{2}{c}{ Mean \pm standard deviation } \\
\cline { 3 - 4 } Study & Year & Case & Control & & Case & Control \\
\hline GSE28423 & 2012 & 19 & 4 & & $4.9500 \pm 1.4447$ & $1.8996 \pm 1.7431$ \\
GSE69524 & 2015 & 10 & 2 & & $4.6094 \pm 0.7349$ & $4.2477 \pm 0.0892$ \\
PMID25352048 & 2015 & 13 & 13 & & $9.5084 \pm 9.1297$ & $2.7254 \pm 2.4619$ \\
\hline
\end{tabular}

$\mathrm{P}<0.05$; standard mean deviation $(95 \%$ confidence interval $)=1.193(0.568-1.819)$; Heterogeneity $\chi^{2}: \mathrm{I}^{2}=26.8 \%$.

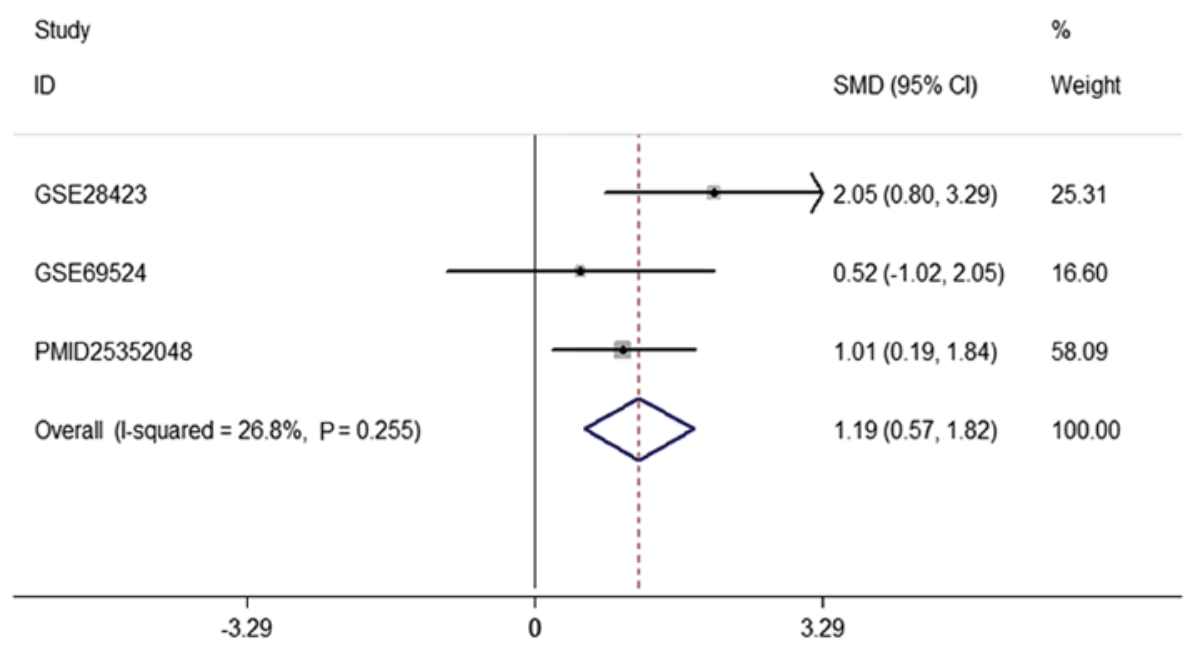

Figure 2. Meta-analysis of miR-542-3p expression in OS. Forest plot of SMDs to confirm the high expression of miR-542-3p in osteosarcoma. miR, microRNA; SMD, standard mean deviation; $\mathrm{CI}$, confidence interval.

if there was significant heterogeneity. Otherwise, a random effects model was used. A Q test based on the $\chi^{2}$ test was used to measure the heterogeneity of study effects distributions. No significant heterogeneity among the studies was considered to exist where the measure of heterogeneity, $\mathrm{I}^{2}$, was demonstrated to be $\leq 50 \%$. A funnel plot was made to evaluate the risk of bias in the included studies. Two-tailed $\mathrm{P}<0.05$ was considered to indicate a statistically significant difference.

\section{Results}

miR-542-3p is upregulated in OS. In order to detect the function of miR-542-3p in OS, two miRNA expression profiles (GSE28423 and GSE69524) and two papers that contained expression data of miR-542-3p in OS were collected (PMID29103020 and PMID25352048, although it was not possible to obtain the expression values of the continuous variable with PMID25352048). The original data were downloaded, from which the expression data of miR-542-3p were extracted, and a continuous variable analysis was subsequently performed using Stata software (Table II). The diagnostic sensitivity and specificity of miR-542-3p in patients with OS are presented in the Forest map (Fig. 2). The pooled sensitivity, specificity, positive likelihood ratio, negative likelihood ratio and diagnostic odds ratio were 0.81 [95\% confidence interval (CI), 0.70-0.89], 0.69 (95\% CI, 0.60-0.77), 2.69 (95\% CI, 2.03-3.57), 0.29 (95\% CI, 0.09-0.94) and 16.13 (95\%
CI, 6.42-40.52), respectively (Fig. 3A-E). The area under the summary receiver operating characteristic (SROC) curve was 0.8657 (Fig. 3F). In addition, the statistical analysis yielded the following values for the listed parameters: $\mathrm{P}=0.255$; standard mean deviation $=1.19 ; 95 \% \mathrm{CI}, 0.57-1.8$; heterogeneity according to the $\chi^{2}$ test, $\mathrm{I}^{2}=26.8 \%$ (Fig. 2). miR-542-3p was identified to be overexpressed in OS tissues and in OS cell lines. As there were fewer than 10 studies, tests for funnel plot asymmetry were not used in the meta-analysis (15).

Prediction of target genes of miR-542-3p. Based on the GEO dataset and the miRWalk2.0 atlas of predicted and experimentally verified miRNA target binding sites, the analysis data of the potential target genes of miR-542-3p were extracted from the gene expression profile of OS. The 12 bioinformatics software packages mentioned above were used to predict the target genes of miR-542-3p, and only those genes that appeared in more than four of the prediction software packages were retained. The overlapping potential target genes are presented in Fig. 4; VANGL2 was an experimental verification gene identified in the literature, and this was also involved in the above analysis.

PPI network analysis. The potential target genes were integrated and analyzed using PPI network analysis. A total of eight hub genes [ubiquitin-60S ribosomal protein L40 (UBA52), Ras-related C3 botulinum toxin substrate (RAC1), 

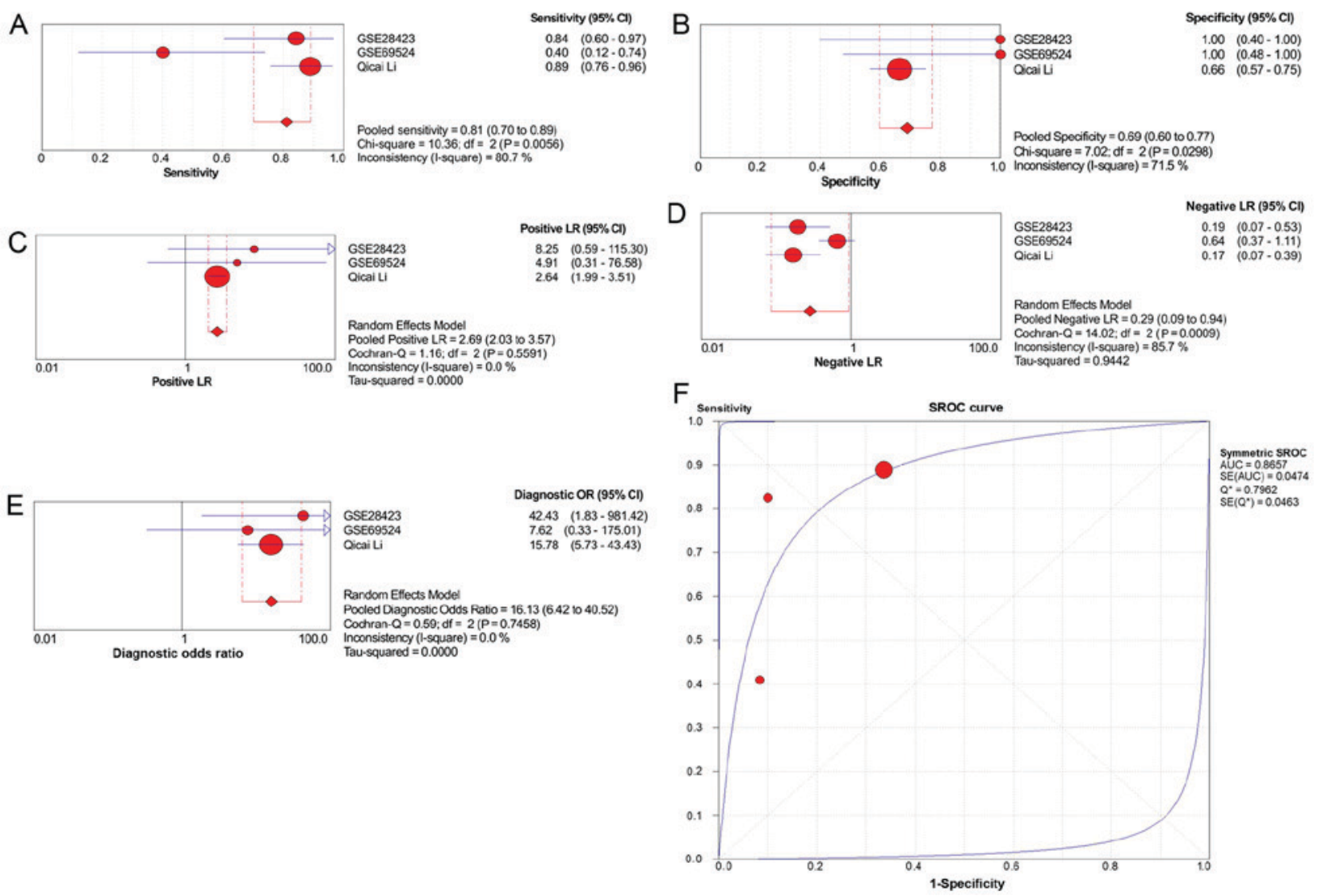

Figure 3. Meta-analysis of miR-542-3p expression in OS. (A) Pooled sensitivity, (B) specificity, (C) positive likelihood ratio, (D) negative likelihood ratio and (E) diagnostic odds ratio parameters were obtained using the software package Stata based on GEO datasets. (F) The combined SROC curve analysis of miR-542-3p is presented. GEO, Gene Expression Omnibus; SROC, summary receiver operating characteristic; CI, confidence interval; AUC, area under the curve; OR, odds ratio.

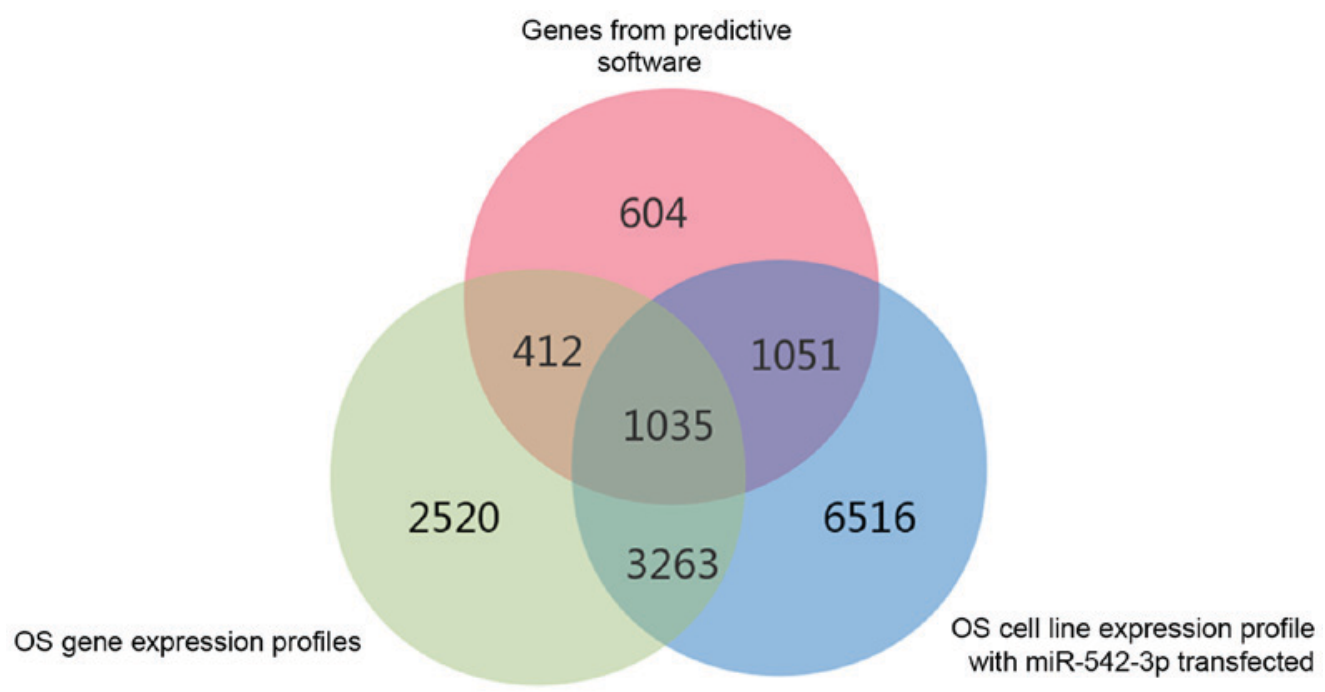

Figure 4. Venn diagram to illustrate the interactions between target genes from predictive software packages and DEGs in OS gene expression profiles, in addition to DEGs in OS cell line expression. DEG, differentially expressed gene; OS, osteosarcoma.

mitogen-activated protein kinase 1 (MAPK1), epidermal growth factor receptor (EGFR), cystic fibrosis transmembrane conductance regulator (CFTR), phosphoinositide 3-kinase regulatory subunit 1 (PI3KR1), AKT serine/threonine kinase 1 (AKT1), and actin-related protein $2 / 3$ complex subunit $1 \mathrm{~A}$ (ARPC1A)] were identified in the PPI network, which were associated with
$>20$ other types of genes. These hub genes were therefore designated as the key target genes of miR-542-3p in OS (Table III).

GO enrichment and KEGG pathway analysis. GO enrichment was used to detect the role of the 1,036 target genes involved the biological process of OS. Three categories were 
Table III. Top ten protein-protein interaction nodes according to STRING.

\begin{tabular}{llcccc}
\hline Node1 & Node2 & $\begin{array}{c}\text { Experimentally determined } \\
\text { interaction }\end{array}$ & $\begin{array}{c}\text { Database } \\
\text { annotated }\end{array}$ & $\begin{array}{c}\text { Automated } \\
\text { text mining }\end{array}$ & $\begin{array}{c}\text { Combined } \\
\text { score }\end{array}$ \\
\hline ACLY & OGDH & 0 & 0.536 & 0.795 & 0.999 \\
ATP6V1A & ATP6V1E1 & 0.932 & 0.9 & 0.826 & 0.999 \\
ATP6V1C1 & ATP6V1E1 & 0.921 & 0.9 & 0.829 & 0.999 \\
UPF2 & UPF1 & 0.965 & 0.9 & 0.98 & 0.999 \\
SEC61A1 & SEC61B & 0.895 & 0.9 & 0.87 & 0.999 \\
AP4E1 & AP4S1 & 0.922 & 0.9 & 0.87 & 0.999 \\
CALM1 & NOS3 & 0.98 & 0.8 & 0.919 & 0.999 \\
RPS27L & RPS23 & 0.973 & 0.9 & 0.144 & 0.999 \\
RANGAP1 & UBE2I & 0.973 & 0.9 & 0.921 & 0.999 \\
FOXO3 & AKT1 & 0.854 & 0.9 & 0.955 & 0.999 \\
\hline
\end{tabular}

included in GO enrichment analysis: The biological process (BP), cellular component (CC), and molecular function (MF) categories. For BPs, the target genes were predominantly associated with $10 \mathrm{GO}$ terms, including 'positive regulation of transcription, DNA-templated', 'intracellular signal transduction' and 'positive regulation of apoptotic process'. Regarding MFs, the target genes were mainly enriched with $10 \mathrm{GO}$ terms, including 'protein binding', 'actin binding' and 'cadherin binding involved in cell-cell adhesion'. For CCs, the target genes were also associated with 10 GO terms, including 'cytoplasm', 'extracellular exosome' and 'cytosol'. The top ten most significant GO enrichment terms are described in Table IV. The KEGG results revealed the signaling pathways of the target genes of miR-542-3p of particular interest, including the insulin $\left(\mathrm{P}=1.04 \times 10^{-5}\right)$, PI3K-AKT $\left(\mathrm{P}=3.17 \times 10^{-5}\right)$, and sphingolipid $\left(\mathrm{P}=3.91 \times 10^{-5}\right)$ signaling pathways. Statistically significant pathways are described in Table V.

\section{Discussion}

Although miR-542-3p was demonstrated to be overexpressed in OS, promoting the proliferation and migration of OS (13), the number of samples used to evaluate miR-542-3p in OS and noncancerous tissues was relatively small, and the molecular mechanisms underlying the role of miR-542-3p in OS remain to be fully elucidated. In the present study, the upregulation of miR-542-3p was verified through continuous variables, and the heterogeneity test revealed slight heterogeneity $\left(\mathrm{I}^{2}<50 \%\right)$. The diagnostic ability of miR-542-3p in OS was investigated by constructing the SROC curve. The area under the curve value of the SROC curve indicated that miR-542-3p may serve as a putative diagnostic target for OS. However, the number of qualifying studies and the sample size of the present study remain a limiting factor, and further studies involving larger sample sizes are required to better evaluate the diagnostic capacity of miR-542-3p in OS.

The present study revealed that miR-542-3p was overexpressed in OS. Li et al (13) also demonstrated that miR-542-3p is highly expressed in OS, and carcinogenicity was increased via targeting of VANGL2. However, lower expression levels of miR-542-3p were identified in numerous other tumor types in previous studies (15-17), and miR-542-3p was reported to exert a tumor-suppressing effect, contrary to what was identified in the present study. Liu et al (16) reported that miR-542-3p is downregulated in non-small cell lung cancer cells, and functions as a tumor suppressor via upregulation of the mitochondrial protein, mitochondrial rRNA methyltransferase 2 . Tao et al (17) demonstrated that miR-542-3p is downregulated in hepatocellular carcinoma tissues, and is a tumor suppressor gene that regulates hepatoma metastasis and epithelialization by targeting ubiquitin protein ligase E3C. Rang et al (18) demonstrated that miR-542-3p is underexpressed in melanoma, and inhibits cellular invasion and metastasis through its action on PIM1 proto-oncogene, serine/threonine kinase. Considered altogether, the previous reports support the notion that overexpression of miR-542-3p may be used as a target for the diagnosis and therapy of OS, although the contrasting results obtained from the various studies of the expression of miR-542-3p, and its role in different types of tumors, merit further study.

Subsequently, 1,035 downregulated target genes were screened from the gene chip of OS and bioinformatics prediction software, also including OS cell line expression profiles with transfected miR-542-3p.

Due to the mutation and methylation of genes, differential expression of genes may arise. High-throughput technology has emerged as an effective method of detecting the expression levels of genes across the entire genome, and this process serves a vital role in identifying abnormal genomic alterations. As a consequence, an increasing number of DEGs associated with OS have been identified through the application of this technology $(19,20)$. Compared with the simple prediction of target genes of miR-542-3p in OS via predictive software, to have combined these data with DEGs from the OS expression chips has undoubtedly resulted in increased specificity. OS cell line expression profiles with transfected miR-542-3p are typically used to simulate the expression of miR-542-3p in OS, in order to identify upregulated or downregulated expression of target genes, with the purpose of investigating the potential role of miR-542-3p, and taking this approach has provided more convincing results in the present study. 
Table IV. GO functional annotation of miR-542-3p target genes.

A, Biological process

\begin{tabular}{|c|c|c|c|c|}
\hline GO ID & GO term & Count & $\%$ & P-value \\
\hline GO:0006605 & Protein targeting & 11 & $6.57 \times 10^{-3}$ & $4.86 \times 10^{-5}$ \\
\hline GO:0035556 & Intracellular signal transduction & 44 & $2.63 \times 10^{-2}$ & $5.76 \times 10^{-5}$ \\
\hline GO:0043065 & Positive regulation of apoptotic process & 35 & $2.09 \times 10^{-2}$ & $1.05 \times 10^{-4}$ \\
\hline GO:0051056 & Regulation of small GTPase mediated signal transduction & 20 & $1.20 \times 10^{-2}$ & $2.15 \times 10^{-4}$ \\
\hline GO:0043401 & Steroid hormone mediated signaling pathway & 12 & $7.17 \times 10^{-3}$ & $3.23 \times 10^{-4}$ \\
\hline GO:0007050 & Cell cycle arrest & 20 & $1.20 \times 10^{-2}$ & $4.17 \times 10^{-4}$ \\
\hline GO:0008286 & Insulin receptor signaling pathway & 14 & $8.37 \times 10^{-3}$ & $4.35 \times 10^{-4}$ \\
\hline GO:0060348 & Bone development & 10 & $5.98 \times 10^{-3}$ & $4.99 \times 10^{-4}$ \\
\hline GO:0045893 & Positive regulation of transcription, DNA-templated & 48 & $2.87 \times 10^{-2}$ & $1.00 \times 10^{-3}$ \\
\hline GO:0060020 & Bergmann glial cell differentiation & 5 & $2.99 \times 10^{-3}$ & $1.05 \times 10^{-3}$ \\
\hline
\end{tabular}

$\mathrm{B}$, Molecular function

\begin{tabular}{|c|c|c|c|c|}
\hline GO ID & GO term & Count & $\%$ & P-value \\
\hline GO:0003779 & Actin binding & 37 & $2.21 \times 10^{-2}$ & $2.75 \times 10^{-6}$ \\
\hline GO:0051117 & ATPase binding & 15 & $8.96 \times 10^{-3}$ & $5.72 \times 10^{-5}$ \\
\hline GO:0003707 & Steroid hormone receptor activity & 12 & $7.17 \times 10^{-3}$ & $2.49 \times 10^{-4}$ \\
\hline GO:0005516 & Calmodulin binding & 23 & $1.37 \times 10^{-2}$ & $1.01 \times 10^{-3}$ \\
\hline GO:0005158 & Insulin receptor binding & 8 & $4.78 \times 10^{-3}$ & $1.42 \times 10^{-3}$ \\
\hline GO:0051015 & Actin filament binding & 17 & $1.02 \times 10^{-2}$ & $3.11 \times 10^{-3}$ \\
\hline GO:0098641 & Cadherin binding involved in cell-cell adhesion & 29 & $1.73 \times 10^{-2}$ & $3.91 \times 10^{-3}$ \\
\hline GO:0008092 & Cytoskeletal protein binding & 9 & $5.38 \times 10^{-3}$ & $5.02 \times 10^{-3}$ \\
\hline GO:0004707 & MAP kinase activity & 5 & $2.99 \times 10^{-3}$ & $6.38 \times 10^{-3}$ \\
\hline
\end{tabular}

C, Cellular component

\begin{tabular}{|c|c|c|c|c|}
\hline GO ID & GO term & Count & $\%$ & P-value \\
\hline GO:0005829 & Cytosol & 255 & $1.52 \times 10^{-1}$ & $1.01 \times 10^{-9}$ \\
\hline GO:0005925 & Focal adhesion & 52 & $3.11 \times 10^{-2}$ & $5.35 \times 10^{-9}$ \\
\hline GO:0005737 & Cytoplasm & 362 & $2.16 \times 10^{-1}$ & $2.13 \times 10^{-8}$ \\
\hline GO:0005789 & Endoplasmic reticulum membrane & 83 & $4.96 \times 10^{-2}$ & $4.33 \times 10^{-7}$ \\
\hline GO:0000139 & Golgi membrane & 61 & $3.65 \times 10^{-2}$ & $2.11 \times 10^{-6}$ \\
\hline GO:0070062 & Extracellular exosome & 206 & $1.23 \times 10^{-1}$ & $2.66 \times 10^{-6}$ \\
\hline GO:0005856 & Cytoskeleton & 43 & $2.57 \times 10^{-2}$ & $5.23 \times 10^{-6}$ \\
\hline GO:0043234 & Protein complex & 44 & $2.63 \times 10^{-2}$ & $3.04 \times 10^{-5}$ \\
\hline GO:0005794 & Golgi apparatus & 76 & $4.54 \times 10^{-2}$ & $3.40 \times 10^{-5}$ \\
\hline
\end{tabular}

Go, gene ontology.

Using the STRING database to identify hub genes, eight loci were eventually screened out: UBA52, RAC1, MAPK1, EGFR, CFTR, PIK3R1, AKT1 and ARPC1A. These genes are likely to be key target genes of miR-542-3p in OS. More detailed information on these hub genes in OS was subsequently sought in the present study.

RAC1 is a small GTPase, which acts as a molecular switch controlling multiple signaling pathways (21). Geng et al (22) identified RAC1 as a direct target gene of miR-224 in OS, and the authors of that study considered that RAC1 to be an oncogene of OS that is associated with the sensitivity of OS to cisplatin. Tan et al (23) demonstrated that chitosan promoted the apoptosis of OS cells by downregulating RAC1.

In a bioinformatics study, Li et al (24) predicted that the MAPK1 gene is the most important gene associated with OS. Their findings were consistent with the results of the present study; however, the role of this gene in OS is yet to be verified experimentally. 
Table V. Top 35 enriched KEGG pathway of target genes.

\begin{tabular}{|c|c|c|c|}
\hline KEGG ID & KEGG term & Count & P-value \\
\hline hsa04910 & Insulin signaling pathway & 24 & $1.04 \times 10^{-5}$ \\
\hline hsa04151 & PI3K-Akt signaling pathway & 42 & $3.17 \times 10^{-5}$ \\
\hline hsa04071 & Sphingolipid signaling pathway & 21 & $3.91 \times 10^{-5}$ \\
\hline hsa04152 & AMPK signaling pathway & 21 & $5.00 \times 10^{-5}$ \\
\hline hsa04510 & Focal adhesion & 29 & $5.95 \times 10^{-5}$ \\
\hline hsa04722 & Neurotrophin signaling pathway & 20 & $1.24 \times 10^{-4}$ \\
\hline hsa05231 & Choline metabolism in cancer & 18 & $1.31 \times 10^{-4}$ \\
\hline hsa05200 & Pathways in cancer & 44 & $1.43 \times 10^{-4}$ \\
\hline hsa04150 & mTOR signaling pathway & 13 & $1.75 \times 10^{-4}$ \\
\hline hsa04919 & Thyroid hormone signaling pathway & 19 & $1.94 \times 10^{-4}$ \\
\hline hsa04022 & cGMP-PKG signaling pathway & 23 & $5.23 \times 10^{-4}$ \\
\hline hsa05212 & Pancreatic cancer & 13 & $5.34 \times 10^{-4}$ \\
\hline hsa04068 & FoxO signaling pathway & 20 & $5.35 \times 10^{-4}$ \\
\hline hsa05205 & Proteoglycans in cancer & 26 & $5.46 \times 10^{-4}$ \\
\hline hsa04931 & Insulin resistance & 17 & $8.94 \times 10^{-4}$ \\
\hline hsa04611 & Platelet activation & 19 & $9.89 \times 10^{-4}$ \\
\hline hsa05213 & Endometrial cancer & 11 & $1.11 \times 10^{-3}$ \\
\hline hsa05160 & Hepatitis C & 19 & $1.29 \times 10^{-3}$ \\
\hline hsa04141 & Protein processing in endoplasmic reticulum & 22 & $1.61 \times 10^{-3}$ \\
\hline hsa05131 & Shigellosis & 12 & $1.67 \times 10^{-3}$ \\
\hline hsa05221 & Acute myeloid leukemia & 11 & $1.99 \times 10^{-3}$ \\
\hline hsa00512 & Mucin type O-Glycan biosynthesis & 8 & $2.35 \times 10^{-3}$ \\
\hline hsa05120 & Epithelial cell signaling in Helicobacter pylori infection & 12 & $2.44 \times 10^{-3}$ \\
\hline hsa05215 & Prostate cancer & 14 & $2.72 \times 10^{-3}$ \\
\hline hsa04664 & Fc epsilon RI signaling pathway & 12 & $2.75 \times 10^{-3}$ \\
\hline hsa04261 & Adrenergic signaling in cardiomyocytes & 19 & $3.69 \times 10^{-3}$ \\
\hline hsa05218 & Melanoma & 12 & $3.89 \times 10^{-3}$ \\
\hline hsa05210 & Colorectal cancer & 11 & $4.33 \times 10^{-3}$ \\
\hline hsa05211 & Renal cell carcinoma & 11 & $6.11 \times 10^{-3}$ \\
\hline hsa04014 & Ras signaling pathway & 25 & $6.27 \times 10^{-3}$ \\
\hline hsa04380 & Osteoclast differentiation & 17 & $6.59 \times 10^{-3}$ \\
\hline hsa05223 & Non-small cell lung cancer & 10 & $6.82 \times 10^{-3}$ \\
\hline hsa04012 & ErbB signaling pathway & 13 & $6.86 \times 10^{-3}$ \\
\hline hsa04066 & HIF-1 signaling pathway & 14 & $6.96 \times 10^{-3}$ \\
\hline hsa04915 & Estrogen signaling pathway & 14 & $7.58 \times 10^{-3}$ \\
\hline
\end{tabular}

KEGG, Kyoto Encyclopedia of Genes and Genomes.

EGFR is a receptor tyrosine kinase that is associated with the pathogenesis of numerous types of cancer (25), modulating the growth, signaling, differentiation, adhesion, migration and survival of cancer cells (26). Hou et al (27) considered that the interaction between transforming growth factor- $\alpha$ and EGFR triggers the activation of PI3K and AKT, which in turn activates nuclear factor- $\kappa \mathrm{B}$, leading to the expression of intercellular adhesion molecule 1 and the promotion of the migration of human OS cells. Zhang et al (28) demonstrated that toosendanin (a triterpenoid extracted from Chinese traditional medicine) inhibited OS by blocking signal transducer and activator of transcription 3 (STAT3) dimerization and impairing the formation of the complex between STAT3 and EGFR.
AKT, also referred to as protein kinase B, exerts numerous roles vital to human physiology and pathology. Counted among its family members are AKT1/PKB $\alpha, \mathrm{AKT} 2 / \mathrm{PKB} \beta$, and AKT3/PKB $\gamma$ (29). Zhu et al (30) demonstrated that the Thr308Ala mutation in AKT1 was able to enhance the cytotoxicity of OS cells induced by the mechanistic target of rapamycin kinase (mTOR) inhibitor, XL388. Han et al (31) reported that tissue inhibitors of metalloproteinase inhibit OS by downregulating AKT1.

Zucchini et al (32) demonstrated that ARPC1A is a molecule that serves a crucial role in the remodeling of the actin cytoskeleton, and CD99 wt (the full-length protein of CD99, a transmembrane protein encoded by the MIC2 gene that is 
involved in multiple cellular events, including cell adhesion and migration) is able to inhibit OS by inhibiting ARPC1A.

Siegel et al (33) identified PIK3R1 as a gene associated with vascular anomalies, forming a link between cancer-associated variants and previously described somatic cell variants of vascular overgrowth syndrome, and the authors of that study hypothesized that PIK3R may be associated with tumors. However, the underlying mechanism of PIK3R in OS requires further investigation, and the other hub genes, including UBA52 and CFTR, have been rarely reported in OS; therefore, similarly, further studies are required to elucidate their underlying mechanisms in OS.

The results of the GO classification enrichment analysis in the present study led to an identification of the possible molecular mechanisms associated with target genes of miR-542-3p in OS, and the GO terms included 'bone development', 'cell cycle arrest' and 'intracellular signal transduction'. Jiang et al (34) confirmed that transmembrane protein 119 contributes to bone development, in addition to the proliferation of OS cells, via the induction of cell cycle arrest at the $\mathrm{G}_{0} / \mathrm{G}_{1}$ phase and apoptosis. Liu et al (35) confirmed that nuclear receptor binding SET domain protein 3 (NSD3) inhibited intracellular signal transduction via the downregulation of a number of genes that had been explored in their previous work, and therefore NSD3 may serve as a potential molecular target for OS therapy. The results of the KEGG pathway analysis in the present study also showed that the target genes of miR-542-3p serve vital roles in OS. Our hypothesis was that the target genes of miR-542-3p may be associated with a number of signaling pathways in order to influence the occurrence and development of OS. Among the 35 significant signaling pathways, the forkhead box O (FOXO), AMP-activated protein kinase (AMPK) and PI3K-Akt signaling pathways have been closely associated with human cancer. FOXO proteins are a subgroup of the FOX superfamily of transcription factors that are able to antagonistically affect the action of insulin and trigger tumor inhibition (36). Evidence has also been published to suggest that inhibiting FOXO1 may promote cell proliferation, enhance colony formation and lead to weak osteogenic differentiation in OS cell lines (37). AMPK is an evolutionarily conserved cellular energy sensor (38). Activation of AMPK inhibits the growth of liver cancer cells and SW620 colorectal cancer cells, in addition to OS cells (39-41). PI3K is an upstream regulator of mTOR, and activation of the AMPK signaling pathway has been demonstrated to be responsible for the transformation of OS cells and the poor prognosis of patients with OS (42). In the present study, a profound enrichment of pathways regulated by osteoclast differentiation was also identified, and this phenomenon is one of the molecular mechanisms that may be involved in the overexpression of miR-542-3p in OS (43).

An increasing amount of evidence has demonstrated that the hub genes of miR-542-3p are directly or indirectly able to regulate the development, occurrence, prognosis, diagnosis and treatment of OS. However, one limitation of the present study was that the association between miR-542-3p and its hub genes was not verified on an experimental level. A large number of these hub genes have been rarely reported to be associated with miR-542-3p in OS. The predicted hub genes identified on the basis of the PPI analysis require further verification via in vivo and in vitro experiments in the future.

In conclusion, the present study confirmed that miR-542-3p is highly expressed in OS, and serves as a tumor promoter.
miR-542-3p exerted its role in promoting OS by regulating target gene networks via specific signaling pathways. The potential target genes and biological functions of miR-542-3p provide novel insights into the DEGs of OS, and miR-542-3p may therefore be a novel target for the early diagnosis and treatment of OS.

\section{Acknowledgements}

Not applicable.

\section{Funding}

The present study was supported by the National Natural Science Foundation of China (grant no. 81560371).

\section{Availability of data and materials}

All data generated or analyzed during this study are included in this published article.

\section{Authors' contributions}

ZL, JNY, WTH, RQH and JM performed the literature searches, data extraction and statistical analyses, and drafted the paper. QJW and GC supervised the literature searches, data extraction and analyses, and reviewed the paper. All authors confirm that they have read and approved the final manuscript.

\section{Ethics approval and consent to participate}

Not applicable.

\section{Patient consent for publication}

Not applicable.

\section{Competing interests}

The authors declare that they have no competing interests.

\section{References}

1. Siegel RL, Miller KD and Jemal A: Cancer statistics, 2017. CA Cancer J Clin 67: 7-30, 2017.

2. Meazza C and Scanagatta P: Metastatic osteosarcoma: A challenging multidisciplinary treatment. Expert Rev Anticancer Ther 16: 543-556, 2016.

3. Venkatramani R, Murray J, Helman L, Meyer W, Hicks MJ, Krance R, Lau C, Jo E and Chintagumpala M: Risk-based therapy for localized osteosarcoma. Pediatr Blood Cancer 63: 412-417, 2016.

4. Zhang Y, Wang Z and Gemeinhart RA: Progress in microRNA delivery. J Control Release 172: 962-974, 2013.

5. Hammond SM: An overview of microRNAs. Adv Drug Deliv Rev 87: 3-14, 2015.

6. Lages E, Ipas H, Guttin A, Nesr H, Berger F and Issartel JP: MicroRNAs: Molecular features and role in cancer. Front Biosci (Landmark Ed) 17: 2508-2540, 2012.

7. Connett P: Water fluoridation: Is fluoride chemophobia? Br Dent J 222: 323-324, 2017.

8. Li Y, Zhang J, Zhang L, Si M, Yin H and Li J: Diallyl trisulfide inhibits proliferation, invasion and angiogenesis of osteosarcoma cells by switching on suppressor microRNAs and inactivating of Notch-1 signaling. Carcinogenesis 34: 1601-1610, 2013. 
9. Althoff K, Lindner S, Odersky A, Mestdagh P, Beckers A Karczewski S, Molenaar JJ, Bohrer A, Knauer S, Speleman F, et al: miR-542-3p exerts tumor suppressive functions in neuroblastoma by downregulating survivin. Int J Cancer 136: 1308-1320, 2015.

10. Cai J, Zhao J, Zhang N, Xu X, Li R, Yi Y, Fang L, Zhang L, Li M, Wu J and Zhang H: MicroRNA-542-3p suppresses tumor cell invasion via targeting AKT pathway in human astrocytoma. J Biol Chem 290: 24678-24688, 2015.

11. Zhang J, Wang S, Han F, Li J, Yu L, Zhou P, Chen Z, Xue S, Dai C and Li Q: MicroRNA-542-3p suppresses cellular proliferation of bladder cancer cells through post-transcriptionally regulating survivin. Gene 579: 146-152, 2016.

12. ShenX,Si Y,YangZ, WangQ,YuanJandZhang X:MicroRNA-542-3p suppresses cell growth of gastric cancer cells via targeting oncogene astrocyte-elevated gene-1. Med Oncol 32: 361, 2015.

13. Li H, Liu H, Pei J, Wang H and Lv H: miR-542-3p overexpression is associated with enhanced osteosarcoma cell proliferation and migration ability by targeting Van Gogh-like 2. Mol Med Rep 11: 851-856, 2015

14. Wang Y, Huang JW, Castella M, Huntsman DG and Taniguchi T: p53 is positively regulated by miR-542-3p. Cancer Res 74 3218-3227, 2014.

15. Sterne JA, Sutton AJ, Ioannidis JP, Terrin N, Jones DR, Lau J, Carpenter J, Rücker G, Harbord RM, Schmid CH, et al: Recommendations for examining and interpreting funnel plot asymmetry in meta-analyses of randomised controlled trials BMJ 343: d4002, 2011.

16. Liu B, Li J, Zheng M, Ge J, Li J and Yu P: MiR-542-3p exerts tumor suppressive functions in non-small cell lung cancer cells by upregulating FTSJ2. Life Sci 188: 87-95, 2017.

17. Tao J, Liu Z, Wang Y, Wang L, Yao B, Li Q, Wang C, Tu K and Liu Q: MiR-542-3p inhibits metastasis and epithelial-mesenchymal transition of hepatocellular carcinoma by targeting UBE3C. Biomed Pharmacother 93: 420-428, 2017.

18. Rang Z, Yang G, Wang YW and Cui F: miR-542-3p suppresses invasion and metastasis by targeting the proto-oncogene serine/threonine protein kinase, PIM1, in melanoma. Biochem Biophys Res Commun 474: 315-320, 2016.

19. Salinas-Souza C, De Andrea C, Bihl M, Kovac M, Pillay N Forshew T, Gutteridge A, Ye H, Amary MF, Tirabosco R, et al: GNAS mutations are not detected in parosteal and low-grade central osteosarcomas. Mod Pathol 28: 1336-1342, 2015.

20. Yang Z, Chen Y, Fu Y, Yang Y, Zhang Y, Chen Y and Li D: Meta-analysis of differentially expressed genes in osteosarcoma based on gene expression data. BMC Med Genet 15: 80, 2014.

21. Seong BK, Lau J, Adderley T, Kee L, Chaukos D, Pienkowska M, Malkin D, Thorner P and Irwin MS: SATB2 enhances migration and invasion in osteosarcoma by regulating genes involved in cytoskeletal organization. Oncogene 34: 3582-3592, 2015

22. Geng S, Gu L, Ju F, Zhang H, Wang Y, Tang H, Bi Z and Yang C: MicroRNA-224 promotes the sensitivity of osteosarcoma cells to cisplatin by targeting Rac1. J Cell Mol Med 20: 1611-1619, 2016.

23. Tan ML, Shao P, Friedhuber AM, van Moorst M, Elahy M, Indumathy S, Dunstan DE, Wei Y and Dass CR: The potential role of free chitosan in bone trauma and bone cancer management. Biomaterials 35: 7828-7838, 2014.

24. Li H, He Y, Hao P and Liu P: Identification of characteristic gene modules of osteosarcoma using bioinformatics analysis indicates the possible molecular pathogenesis. Mol Med Rep 15: 2113-2119, 2017.

25. Kovacs E, Zorn JA, Huang Y, Barros T and Kuriyan J: A structural perspective on the regulation of the epidermal growth factor receptor. Annu Rev Biochem 84: 739-764, 2015.

26. Biscaglia F, Rajendran S, Conflitti P, Benna C, Sommaggio R, Litti L, Mocellin S, Bocchinfuso G, Rosato A, Palleschi A, et al: Enhanced EGFR targeting activity of plasmonic nanostructures with engineered GE11 peptide. Adv Healthc Mater: 6, 2017 doi: 10.1002/adhm.201700596.

27. Hou CH, Lin FL, Tong KB, Hou SM and Liu JF: Transforming growth factor alpha promotes osteosarcoma metastasis by ICAM-1 and PI3K/Akt signaling pathway. Biochem Pharmacol 89: 453-463, 2014.
28. Zhang T, Li J, Yin F, Lin B, Wang Z, Xu J, Wang H, Zuo D, Wang G, Hua $Y$ and Cai Z: Toosendanin demonstrates promising antitumor efficacy in osteosarcoma by targeting STAT3. Oncogene 36: 6627-6639, 2017.

29. Tang F, Wang Y, Hemmings BA, Rüegg C and Xue G: $\mathrm{PKB} / \mathrm{Akt}$-dependent regulation of inflammation in cancer. Semin Cancer Biol 48: 62-69, 2018.

30. Zhu YR, Zhou XZ, Zhu LQ, Yao C, Fang JF, Zhou F, Deng XW and Zhang YQ: The anti-cancer activity of the mTORC1/2 dual inhibitor XL388 in preclinical osteosarcoma models. Oncotarget 7: 49527-49538, 2016.

31. Han XG, Li Y, Mo HM, Li K, Lin D, Zhao CQ, Zhao J and Tang TT: TIMP3 regulates osteosarcoma cell migration, invasion, and chemotherapeutic resistances. Tumour Biol 37: 8857-8867, 2016

32. Zucchini C, Manara MC, Pinca RS, De Sanctis P, Guerzoni C, Sciandra M, Lollini PL, Cenacchi G, Picci P, Valvassori L and Scotlandi K: CD99 suppresses osteosarcoma cell migration through inhibition of ROCK2 activity. Oncogene 33: 1912-1921, 2014.

33. Siegel DH, Cottrell CE, Streicher JL, Schilter KF, Basel DG, Baselga E, Burrows PE, Ciliberto HM, Vigh-Conrad KA, Eichenfield LF, et al: Analyzing the genetic spectrum of vascular anomalies with overgrowth via cancer genomics. J Invest Dermatol 138: 957-967, 2018.

34. Jiang ZH, Peng J, Yang HL, Fu XL, Wang JZ, Liu L, Jiang JN, Tan YF and Ge ZJ: Upregulation and biological function of transmembrane protein 119 in osteosarcoma. Exp Mol Med 49: e329, 2017.

35. Liu Z, Piao L, Zhuang M, Qiu X, Xu X, Zhang D, Liu M and Ren D: Silencing of histone methyltransferase NSD3 reduces cell viability in osteosarcoma with induction of apoptosis. Oncol Rep 38: 2796-2802, 2017.

36. Guzman-Perez V, Bumke-Vogt C, Schreiner M, Mewis I, Borchert A and Pfeiffer AF: Benzylglucosinolate derived isothiocyanate from tropaeolum majus reduces gluconeogenic gene and protein expression in human cells. PLoS One 11: e0162397, 2016

37. Guan H, Tan P, Xie L, Mi B, Fang Z, Li J, Yue J, Liao H and Li F: FOXO1 inhibits osteosarcoma oncogenesis via Wnt/ $\beta$-catenin pathway suppression. Oncogenesis 4: e166, 2015.

38. Dasgupta B and Chhipa RR: Evolving lessons on the complex role of AMPK in normal physiology and cancer. Trends Pharmacol Sci 37: 192-206, 2016.

39. Zheng L, Yang W, Wu F, Wang C, Yu L, Tang L, Qiu B, Li Y, Guo L, Wu M, et al: Prognostic significance of AMPK activation and therapeutic effects of metformin in hepatocellular carcinoma. Clin Cancer Res 19: 5372-5380, 2013.

40. Cho SY, Lee HJ, Lee HJ, Jung DB, Kim H, Sohn EJ, Kim B, Jung JH, Kwon BM and Kim SH: Activation of AMP-activated protein kinase $\alpha$ and extracelluar signal-regulated kinase mediates CB-PIC-induced apoptosis in hypoxic SW620 colorectal cancer cells. Evid Based Complement Alternat Med 2013: 974313, 2013

41. Zhao Z, Yin JQ, Wu MS, Song G, Xie XB, Zou C, Tang Q, Wu Y, Lu J, Wang Y, et al: Dihydromyricetin activates AMP-activated protein kinase and P38(MAPK) exerting antitumor potential in osteosarcoma. Cancer Prev Res (Phila) 7: 927-938, 2014.

42. Hu K, Dai HB and Qiu ZL: mTOR signaling in osteosarcoma: Oncogenesis and therapeutic aspects (Review). Oncol Rep 36: 1219-1225, 2016.

43. Kureel J, Dixit M, Tyagi AM, Mansoori MN, Srivastava K, Raghuvanshi A, Maurya R, Trivedi R, Goel A and Singh D: miR-542-3p suppresses osteoblast cell proliferation and differentiation, targets BMP-7 signaling and inhibits bone formation. Cell Death Dis 5: e1050, 2014.

This work is licensed under a Creative Commons Attribution-NonCommercial-NoDerivatives 4.0 International (CC BY-NC-ND 4.0) License. 